\title{
Measuring the Masses of Young Stars
}

\author{
Michal Simon \\ Dept. of Physics and Astronomy, SUNY, Stony Brook, NY, \\ 17794-3800, USA
}

\begin{abstract}
This paper presents the masses of young stars measured by the rotation of their circumstellar disks (Simon, Dutrey, and Guilloteau 2000). Their precision is good enough to enable meaningful tests of theoretical models of pre-main sequence evolution. The tests are however limited by the imprecision with which the distances to the stars are known. The astrometric instruments now being developed have the potential to remove this limitation.
\end{abstract}

\section{Introduction}

The participants in this conference know well that mass is the most important property of a star. Unfortunately, below a solar mass, our knowledge of accurate stellar masses is still very limited. The result is that the inputs essential to theories of star formation such as the ages of stars in a star forming region (SFR), its star-forming history, the mass spectrum of the stars produced, and the distribution of masses in binaries, are imprecisely known.

The mass and age of a pre-main sequence (PMS) star are usually estimated from its location in the HR-diagram relative to theoretical models of its evolution. The uncertainties in measuring the luminosity and effective temperature of a PMS star, and the uncertainties in the tracks, particularly below $\sim 1.0 \mathrm{M}_{\odot}$, limit the reliability of the mass and age estimates. For example, for a typical point in the $\mathrm{HR}$ diagram corresponding to a $\mathrm{K} 7$ star with $\mathrm{L}=1.0 \mathrm{~L}_{\odot}$, Table 1 shows that the tracks yield masses and ages discrepant by factors of 2 to 3 .

Table 1. Theoretical Mass and Age Estimates for a $\mathrm{K} 7, \mathrm{~L}=1.0 \mathrm{~L}_{\odot}$ PMS Star

\begin{tabular}{|l|cc|}
\hline \hline Calculation & $\mathrm{M} / \mathrm{M}_{\odot}$ & Age (Myr) \\
\hline Swenson et al. (1994) & 0.65 & 1.0 \\
D'Antona and Mazzitelli(1997) & 0.45 & 0.8 \\
Baraffe et al. (1998) & 0.80 & 1.0 \\
Palla and Stahler (1999) & 0.80 & 2.0 \\
Siess et al. (2000) & 0.78 & 3.0 \\
\hline
\end{tabular}

In the absence of measurements of the mass or age of a star, astronomers have applied other tests of the PMS tracks. Hartigan et al. (1994), Casey et 
al. (1998), Luhman (1999) and White et al. (1999) investigated whether the tracks yield the same ages for stars thought to be coeval and found significant differences in the extent to which the available tracks satisfied this requirement.

The situation is improving rapidly. We now know the masses of a few (5-10), young $(1-10 \mathrm{Myr})$, low mass $\left(<1 \mathrm{M}_{\odot}\right)$ stars to an internal precision of a few per cent. Some of these results have been reported in posters at this conference. These new measurements make it possible to test the accuracy of the theoretical PMS tracks. Often the absolute precision of the measured mass remains limited by the precision to which the distance is known. As the Golden Age of Astrometry approaches, the prospects are excellent to increase the numbers of stars with accurate masses, to enlarge the mass range over which they are known, and to measure their distances with high precision.

\section{Dynamical Methods of Mass Measurement}

Methods based on motion under the influence of gravity provide the only reliable determinations of mass. These include mapping the orbits of resolved binaries, historically the visual binaries (VBs), determining the velocity vs. phase of spectroscopic binaries (SBs), several hybrid methods, and of particular applicability to young stars, mapping the rotation of circumstellar disks by mm-wave interferometry. Table 2 summarizes the parameters derivable from the VBs and SBs (e.g. Heintz 1978).

Table 2. Binary Parameters Derivable When Distance Is Not Known

\begin{tabular}{|l||cccc|l|}
\hline \hline & $\begin{array}{c}\text { Visual Bin. } \\
\text { (VB) }\end{array}$ & $\begin{array}{c}\text { Single-lined } \\
\text { Spec. Bin. (SB1) }\end{array}$ & $\begin{array}{c}\text { Double-lined } \\
\text { Spec. Bin. (SB2) }\end{array}$ & $\begin{array}{c}\text { Eclipsing } \\
\text { (SB2) }\end{array}$ & Notes \\
\hline$P$ & $\mathrm{Y}$ & $\mathrm{Y}$ & $\mathrm{Y}$ & $\mathrm{Y}$ & \multirow{2}{*}{} \\
$a$ & $\mathrm{Y}$ & $a_{1} \sin i$ & $a \sin i$ & $\mathrm{Y}$ & 1,2 \\
$e$ & $\mathrm{Y}$ & $\mathrm{Y}$ & $\mathrm{Y}$ & $\mathrm{Y}$ & \\
$i$ & $\mathrm{Y}$ & $\mathrm{N}$ & $\mathrm{N}$ & $\mathrm{Y}$ & \multirow{2}{*}{} \\
$\mathrm{M}$ & $\mathrm{N}$ & $f(M)$ & $M_{1} \sin ^{3} i$ and & $M_{1}$ and & 2,3 \\
& & & $M_{2} \sin ^{3} i$ & $M_{2}$ & \\
\hline
\end{tabular}

Parameters: $P=$ period, $a=$ semi-major axis, $e=$ eccentricity, $i=$ inclination, and $M=$ mass. Notes: 1) For VB in arc sec, for SBs in physical units. 2) If motion of VB around barycenter is measured, then $a_{1}$ and $a_{2}$ are known and hence $M_{1}$ and $M_{2}$. 3) Mass function, $f(M)=\left(M_{2} \sin i\right)^{3} /\left(M_{1}+M_{2}\right)^{2}$

Since masses of the VBs are derived from Kepler's 3rd Law they scale as $D^{3}$. Stellar masses measured using the rotation of the associated circumstellar disks depend on the radial distance in the disk so scale directly with distance. The uncertainties in the measured masses, $\Delta M / M$, resulting from the distance uncertainty are therefore $3 \Delta D / D$ or $\Delta D / D$, depending on the approach. These can be significant. The Taurus SFR, for example extends $\sim 15^{\circ}$ on the sky. If its depth is comparable to its width, the distance to a specific star can scatter by $\pm 20 \mathrm{pc}$ around the $140 \mathrm{pc}$ average distance to the SFR (Kenyon et al. 1994), an 
uncertainty of $\pm 14 \%$. The techniques used to measure mass can achieve much lower uncertainties so their over-all accuracy is compromised by the uncertainty in the distance to a particular star. The precision, $\sim 1$ mas, and sensitivity, V $\sim 9 \mathrm{mag}$, of HIPPARCOS were insufficient to remove this limitation.

SBs do not provide the orbital inclination $i$. In the SB1s, the two masses and $i$ are inextricably linked in the mass function. In the SB2s, the mass ratio is known but the determination of their values separately requires $i$. Various "tricks" and hybrid methods are used to overcome this limitation. The best known trick is that of the eclipsing double-lined spectroscopic binaries (ESB2s) in which $i \sim 90^{\circ}$. Combining the parameters of an SB with orbit measurement can yield the masses since a VB yields $i$ (e.g. Torres et al. 1997, Koresko et al. 1998, and Hummel 2000). The development of optical and infrared interferometry over baselines of 10's of meters has enabled mass measurements of bright binaries by this hybrid technique. Interferometers now under construction at the Keck and VLT observatories, and those planned for space platforms, will have the sensitivity to observe the nearby young stars.

\section{Application to Young Visual Binaries and Spectroscopic Binaries}

Mapping the orbits of VBs among the young stars is being pursued by groundbased techniques (Ghez et al. 1995 and Thiébaut et al. 1995) and the fine guidance sensors (FGS) aboard the HST (Simon et al. 1996). The smallest separations resolvable with high precision are 10-20 milliarc sec (mas), corresponding to $1.5-3 \mathrm{AU}$ at the distance of the Taurus SFR. For binaries with total mass $\sim 1 \mathrm{M}_{\odot}$, this corresponds to orbital periods $>$ a few years. With one exception (see below), observations of VBs have not yet yielded masses because most of the binaries being followed seem to have periods measured in decades.

Young SB1s and SB2s have also been identified (e.g. Mathieu 1994) and discoveries of several were announced at this conference (Reipurth et al. 2000, Quast et al. 2000, and Torres et al. 2000). The ESB2s are rare, of course. Prior to this conference, only two PMS ESB2s, TY CrA and RS Cha AB, both with primary and secondary masses greater than $1.5 \mathrm{M}_{\odot}$ were known (Casey et al. 1998 and Mamajek et al. 2000). At this conference, Covino et al. (2000) reported the discovery of the ESB2 $R X J 0529.4+0041$ (period = 3.04d) with primary and secondary masses $1.30 \pm 0.03$ and $0.95 \pm 0.03 \mathrm{M}_{\odot}$. Plotting the components in an HR diagram, they find that the primary is reasonably consistent with the tracks of D'Antona and Mazzitelli (1994), Palla and Stahler (1993), and Swenson et al. (1994), but that the secondary is consistent only with those of Swenson et al. The secondary is also inconsistent with the more recent tracks of Baraffe et al. (1998, BCAH), Palla and Stahler (1999, PS), and Siess et al. (2000, SDF). Since mass measurements of single stars in the mass range $1-0.5 \mathrm{M}_{\odot}$ $(\S 4)$, agree reasonably well with the BCAH, PS, and SDF tracks, one wonders whether models of single stars are applicable to a pair as close and distorted as the components of $R X J 0529.4+0041$ must be (see also Quast et al. 2000).

Steffen et al. (2000) combined an astrometric orbit measured by the FGS of the $H S T$ with the spectroscopic solution for the SB2 NTTS $045251+3016$ to determine the primary and secondary masses, $1.43 \pm 0.34$ and $0.78 \pm 0.20 \mathrm{M}_{\odot}$, respectively. This result involved a double hybrid technique; the NTTS was 
converted from a visible light SB1 to an SB2 by IR spectroscopy (Prato 1998, Mazeh et al. 2000). The precision of the mass measurement is sufficient to indicate that the $\mathrm{BCAH}$ tracks best fit the observations.

\section{Masses of Stars with Circumstellar Disks}

The resolvable disks of PMS stars offer another dynamical technique, that of mapping their rotation and hence measuring the central mass (Dutrey et al. 1994, Mannings and Sargent 1997, Guilloteau and Dutrey 1998). The results presented here are based on ${ }^{12} \mathrm{CO} J=2-1$ line observations at the IRAM Plateau de Bure interferometer obtained with A. Dutrey and S. Guilloteau and described more fully in our paper (Simon et al. 2000). Table 3 lists our results for PMS stars in the Taurus SFR. We ignore the mass of the disk because it is usually only a few per cent that of the star. Even in GG Tau A, which probably has the most massive disk of the stars in our sample, the mass of the circumbinary disk is $\sim 10 \%$ that of the stars within it (Guilloteau et al. 1999). In the Table, $i_{C O}$ is the disk inclination measured from the $\mathrm{CO}$ line maps, $V_{100} \sin i$ is the projected disk velocity $(\mathrm{km} / \mathrm{s})$ at reference radius $100 \mathrm{AU}$, and $v$ is the exponent of the rotation velocity law, $V(r) \propto r^{-v} ; v=0.50$ indicates Keplerian rotation. The internal precision of the masses is limited mostly by the uncertainty in the inclination. For disks with $i_{C O}>35^{\circ}$, the internal precision of the mass is better than $6 \%$. The absolute masses are, however, not as well known because of the uncertainty in the actual distance.

Table 3. Dynamic Masses from Disk Rotation $(D=140 \mathrm{pc})$

\begin{tabular}{|l||l|r|c|c|}
\hline \hline & $i_{C O}$ & $V_{100} \sin (i)$ & \multicolumn{1}{c|}{$-v$} & $\mathrm{M}_{*} / \mathrm{M}_{\odot}$ \\
\hline Singles: & & & & \\
MWC 480 & $+38 \pm 1$ & $-2.38 \pm 0.02$ & $0.50 \pm 0.02$ & $1.65 \pm 0.07$ \\
LkCa15 & $+52 \pm 1$ & $-2.30 \pm 0.02$ & $0.56 \pm 0.03$ & $0.97 \pm 0.03$ \\
DL Tau & $+35 \pm 2$ & $1.90 \pm 0.06$ & $0.55 \pm 0.03$ & $0.72 \pm 0.11$ \\
GM Aur & $+56 \pm 2$ & $2.30 \pm 0.08$ & $0.5 \pm 0.1$ & $0.84 \pm 0.05$ \\
DM Tau & $-32 \pm 1$ & $-1.17 \pm 0.02$ & $0.53 \pm 0.01$ & $0.55 \pm 0.03$ \\
CY Tau & $+30 \pm 10$ & $-1.10 \pm 0.10$ & $0.50 \pm 0.08$ & $0.55 \pm 0.33$ \\
BP Tau & $+30_{-2}^{+4}$ & $1.67 \pm 0.06$ & $0.54 \pm 0.07$ & $1.24_{-0.32}^{0.25}$ \\
\hline Binaries: & & & & \\
GG Tau A & $+37 \pm 1$ & $2.05 \pm 0.06$ & $0.5 \pm 0.1$ & $1.28 \pm 0.07$ \\
UZTau E & $-56 \pm 2$ & $-2.83 \pm 0.05$ & $0.53 \pm 0.03$ & $1.31 \pm 0.08$ \\
\hline
\end{tabular}

Comparison of these mass measurements with theoretical calculations of PMS tracks in an HR diagram provides detailed examples of the differences among the calculated tracks and illustrates the accuracy required for meaningful tests of the tracks. We use spectral types, conversion of spectral type to $T_{\text {eff }}$ appropriate to the main sequence and stellar luminosity, $L_{*}$, evaluated at $\mathrm{D}=140$ pc, as provided by Kenyon and Hartmann (1995). The HR diagram in Fig. 1a shows excerpts from PMS tracks calculated by DM97, BCAH, PS99, and SDF. 


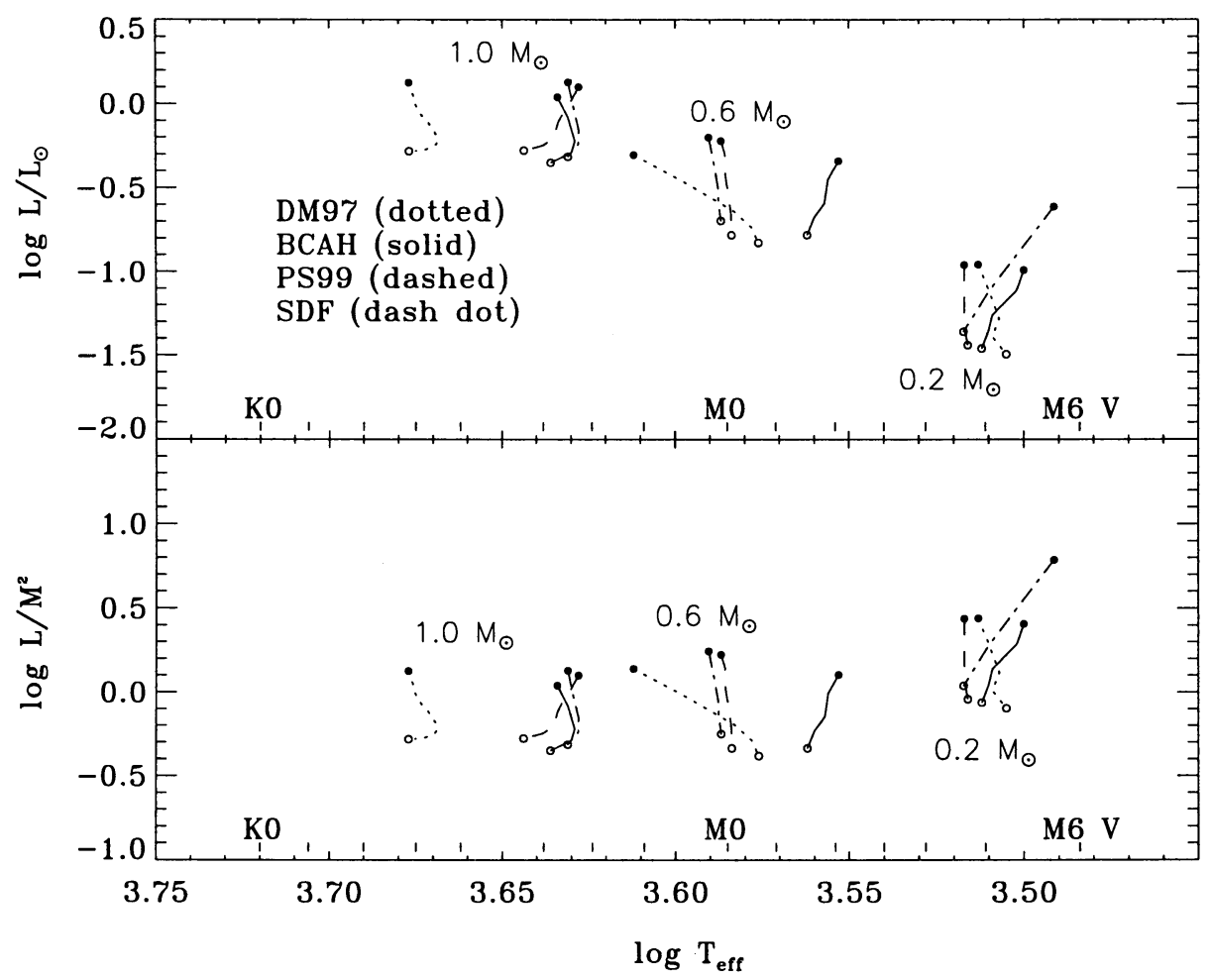

Figure 1. Top: An HR diagram showing theoretical evolutionary tracks for stars of mass $0.2,0.6$, and $1.0 \mathrm{M}_{\odot}$ calculated by DM97, BCAH, PS99, and SDF between the ages 2 Myr (filled circles) and 10 Myr (open circles). Bottom: The same tracks as in upper panel plotted as $L / M^{2}$ vs $T_{\text {eff }}$ (see text).

The BCAH tracks for $\mathrm{M} / \mathrm{M}_{\odot}<0.7$ are for mixing length parameter (mixing length/pressure scale height) $=1.0$ and 1.9 for $\mathrm{M} / \mathrm{M}_{\odot} \geq 0.7$ (BCAH and Baraffe, priv. comm.). Differences among the theoretical calculations for a star of given age and mass are obvious. For example, the DM97 tracks are hotter than those of BCAH for $M_{*} \sim 0.2 M_{\odot}$. SDF's models at 0.2 and $0.1 \mathrm{M}_{\odot}$ appear to contract more slowly than those of DM97, BCAH, and PS99. The differences among the tracks arise in different treatments of stellar convection, the equation of state, opacities, and stellar atmospheres. Each of these becomes important in specific regions of the HR diagram, so comprehensive tests of the theoretical tracks will require accurate data over as wide a range of mass and age as possible.

Since $L_{*} \propto D^{2}$, and $M_{*} \propto D$ for masses determined by disk rotation, the distance uncertainty affects a star's location in the HR diagram. We therefore use modified HR diagrams in which the distance-independent parameter $L / M^{2}$ is plotted versus $T_{\text {eff }}$. Fig. 1b compares the tracks on this basis. 
Fig. 2 compares the masses of singles in Table 3 with PMS tracks calculated by DM97, BCAH, PS99, and SDF. The uncertainties along the $\mathrm{T}_{\text {eff }}$ axis are \pm 1 spectral type and along the vertical axis, the internal uncertainties in mass and an assumed $\pm 10 \%$ uncertainty in the luminosity. In this presentation, if a star does not lie on the track corresponding to its measured mass, either its distance is not the average value, $140 \mathrm{pc}$, or the theoretical track is wrong.

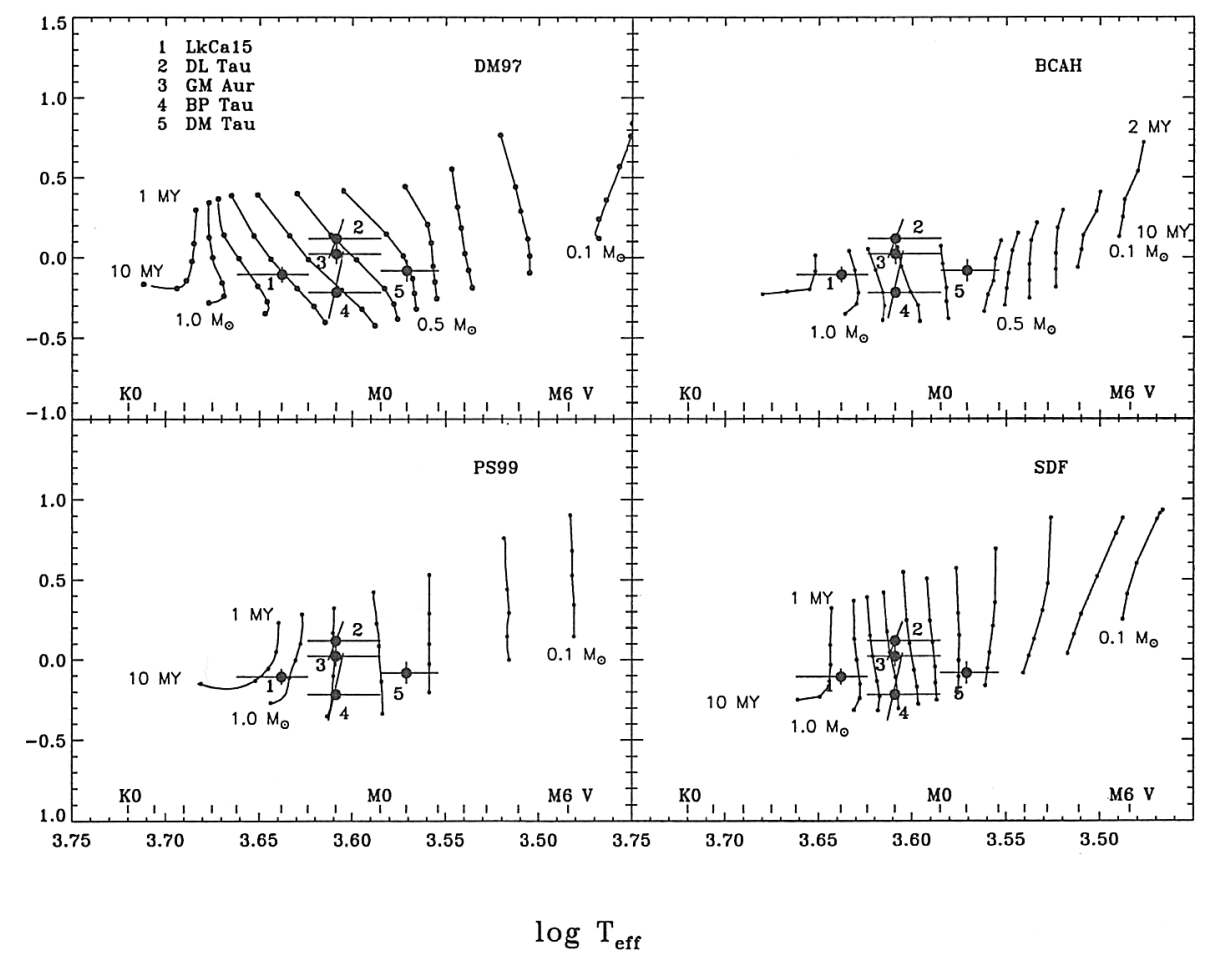

Figure 2. Upper Left: The results for the single T Tauri stars plotted as $L / M^{2}$ vs $T_{\text {eff }}$ for theoretical evolutionary tracks for stars of mass $0.1,0.2,0.3,0.4,0.5,0.6,0.7,0.8,0.9,1.0$ and $1.2 \mathrm{M}_{\odot}$ calculated by DM97. The filled dots are at ages 1,2,3, 5, 7, and 10 Myr. Upper Right: Same, as but for tracks calculated by BCAH. Here the indicated ages are 2, 3, 5, 7, and 10 Myr. Lower Left: Same for tracks calculated by PS99 at masses $0.1,0.2,0.4,0.6,0.8,1.0$, and $1.2 \mathrm{M}_{\odot}$. The indicated ages 1, 2, 3, 5, and 10 Myr.Lower Right: Same for tracks calculated by $\mathrm{SDF}$. The range of masses and indicated ages are same as for DM97.

The $L / M^{2}$ value for for $L k C a 15$, for example, lies near the $1.0 \mathrm{M}_{\odot}$ tracks calculated by BCAH, PS99, and SDF consistent with its mass $0.97 \pm 0.03 \mathrm{M}_{\odot}$ at $140 \mathrm{pc}$ distance. These tracks yield a consistent age estimate of 3-5 Myr. However, the $L / M^{2}$ value lies on the $0.8 \mathrm{M}_{\odot}$ track calculated by DM97. Agreement with the DM97 track would require a distance of $\sim 115 \mathrm{pc}$ but would not affect the age estimate. Similarly, for GM Aur, the $L / M^{2}$ value relative to the BCAH, 
PS99, and SDF tracks is consistent with its mass $0.84 \pm 0.05 \mathrm{M}_{\odot}$ at $140 \mathrm{pc}$. The tracks provide an age estimate of $\sim 3 \mathrm{Myr}$. However, the $L / M^{2}$ value lies close to DM97's $0.6 \mathrm{M}_{\odot}$ track which would require a distance of $103 \mathrm{pc}$ to force agreement with the dynamical mass. At the lower masses, the $L / M^{2}$ values of DL Tau and DM Tau are consistent with the four sets of tracks.

BP Tau's $L / M^{2}$ values lies between DM97's 0.6 and $0.8 \mathrm{M}_{\odot}$ tracks, and close to BCAH, PS99, and SDF's $0.8 \mathrm{M}_{\odot}$ tracks, while our measured mass at $140 \mathrm{pc}$ is in the range 0.92 to $1.49 \mathrm{M}_{\odot}$. This suggests that the distance to $\mathrm{BP}$ Tau may be closer than $140 \mathrm{pc}$ but not as extreme as the HIPPARCOS value, $53_{-11}^{+17}$ pc (Favata et al. 1998). The large uncertainty in the mass produces a large spread in the age estimate, 2-10 Myr.

Fig. 3 compares the dynamical mass of the HAeBe star MWC480 with the DM97, PS99, and SDF tracks. We calculated the stellar luminosity, $11.5 \mathrm{~L}_{\odot}$, using the spectral energy distribution derived by Malfait et al. (1998) evaluated at $\mathrm{D}=140 \mathrm{pc}$ and plotted it at spectral type A4 estimated by Grady (1999) from HST spectra. MWC480's $L / M^{2}$ value lies close to the $2.0 \mathrm{M}_{\odot}$ tracks for the three calculations at an age $\sim 7 \mathrm{Myr}$. The mass at $140 \mathrm{pc}$ distance $1.65 \pm 0.07$ $\mathrm{M}_{\odot}$ suggests that MWC 480 lies at a somewhat greater distance. A distance of $170 \mathrm{pc}$ would yield a dynamical mass of $2.0 \mathrm{M}_{\odot}$ and would be within $2 \sigma$ of the HIPPARCOS measurement of $131_{-18}^{+24}$ pc (van den Ancker et al. 1998).

\section{Summary and Future Work}

The present situation is that:

1) Measurements of PMS masses are becoming available with high enough precision that direct tests of calculations of PMS evolution are possible.

2) The BCAH, PS99, and SDF models are in reasonable agreement with the measured dynamical masses at the average distance to the Taurus SFR. To force agreement between the DM97 models and mass measurements for stars in the $\sim 0.7$ to $1 \mathrm{M}_{\odot}$ range (Lk Ca 15, and GM Aur) would require that these stars lie at unacceptably near distances.

3) The position of the HAeBe star MWC 480 with respect to tracks suggests it lies on the far side of the Taurus SFR at $\sim 170$ pc.

For masses measured by disk rotation, tests of the PMS tracks are limited by our present ignorance of actual distances to individual stars. The examples in the previous section show meaningful tests of tracks require absolute uncertainty of the measured mass less than $\sim 5 \%$. Since the internal precision of the mass measurement better than this is attainable now, we should require that the distance measurement not compromise the overall uncertainty. The astrometric missions now planned or in construction (e.g. FAME, GAIA, and SIM) have the potential to provide distances with sufficient precision. The proposed capabilities of SIM (http://sim.jpl.nasa.gov) translate to parallaxes with better than $1 \%$ precision for PMS stars in the Orion SFR.

So far, measurements by the CS disk technique pertain to stars with $M>$ $0.5 \mathrm{M}_{\odot}$ so we have been unable to test the models at the lowest end of the stellar mass spectrum (but, see White et al. 1999). This is a serious limitation and results, at least in part, from the range of masses of stars formed in the Taurus 


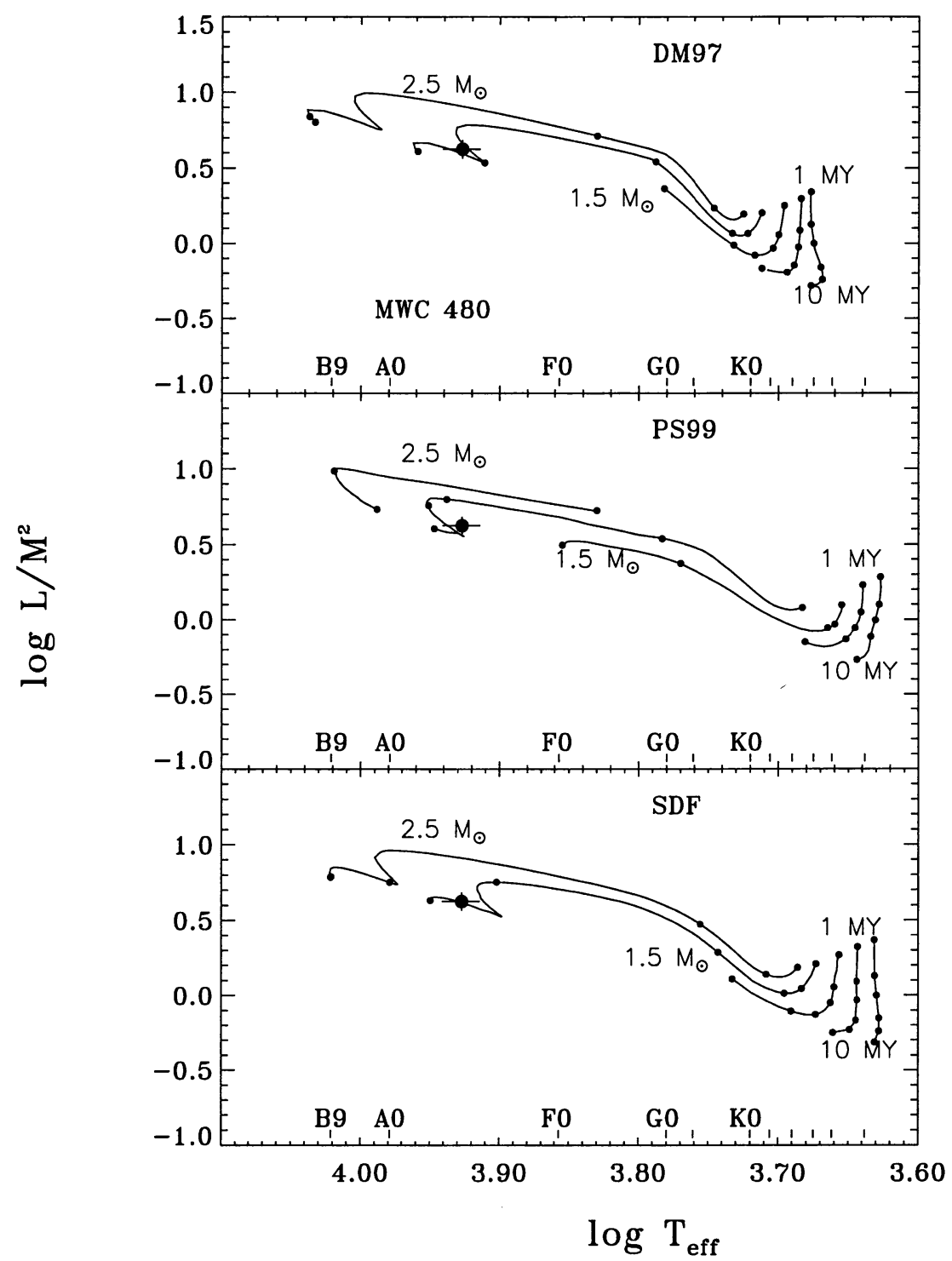

Figure 3. Top: $L / M^{2}$ vs $T_{\text {eff }}$ for the HAeBe star MWC480 plotted with DM97 tracks for stars of mass 1.0, 1.2, 1.5, 2.0 and $2.5 \mathrm{M}_{\odot}$. The dots indicate ages $1,2,3,5,7$, and $10 \mathrm{Myr}$. For the $2.5 \mathrm{M}_{\odot}$ track, the isochrone dots stop at $7 \mathrm{Myr}$ at this age the star is on the main sequence.Middle: Same but for tracks calculated by PS99. The indicated ages are $1,2,3,5$, and $10 \mathrm{Myr}$. The $2.5 \mathrm{M}_{\odot}$ star is on the main sequence at $5 \mathrm{Myr}$. Bottom: Same but for SDF tracks. The range of masses and indicated ages are same as for DM97. 
SFR. We look forward to the capabilities of the ALMA mm-wave interferometric array which will open the rich Orion SFR to investigation. Conversion of the observationally derived parameter, spectral type, to that provided by the models, $T_{\text {eff }}$, is also a source of uncertainty, particularly at the lowest masses. Since stars contracting to the main sequence represent a range of surface gravities, the conversion may depend on age. Relief from this problem should become available soon as the model calculations which include stellar atmospheres provide diagnostics such as color indices and model spectra, for closer comparison with the observations. The prospects are excellent for rapid progress on these problems in the next decade.

Acknowledgments. It is a pleasure to acknowledge my fruitful collaboration with Anne Dutrey and Stephane Guilloteau and to thank Russel White for comments on a draft of this paper. This work was supported in part by NSF Grant 98-19694.

\section{References}

Baraffe, I. et al. 1998, A\&A, 337, 403 (BCAH)

Casey, B. W. et al. 1998, AJ, 115, 1617

Covino, E. et al. 2000, IAU 200 Poster Proc. ${ }^{1}, 9$

D'Antona F. Mazzitelli, I. 1994, ApJS, 90, 467

D'Antona F. \& Mazzitelli, I. 1997, Mem. S. A. It., 68, 807

Dutrey, A., Guilloteau, S., \& Simon, M. 1994, A\&A 286, 149

Dutrey, A. et al. 1998, A\&A, 338, L63

Favata, F. et al. 1998, A\&A, 335, 218

Ghez, A. M. et al. 1995, AJ, 10, 753

Grady, C. 1999, priv. comm.

Guilloteau, S. \& Dutrey, A. 1998, A\&A, 339, 467

Guilloteau, S., Dutrey, A, \& Simon, M. 1999, A\&A, 348, 570

Hartigan, P., Strom, K. M., \& Strom, S. E. 1994, ApJ, 452, 736

Heintz, W. D. 1978, Double Stars, (Dordrecht:Reidel)

Hummel, C. A. 2000, IAU 200 Poster Proc. ${ }^{1}, 154$

Kenyon, S. J., Dobryzycka, D., \& Hartmann, L. 1994, AJ, 108, 1872

Kenyon, S. J., \& Hartmann, L. 1995, ApJS, 101, 117

Koresko, C. D. et al. 1998, ApJ, 509, L45

Luhman, K. L. 1999, ApJ 525, 466

Malfait, K. et al. 1998, A\&A 331, 211

Mamajek, E. E., Lawson, W. A., \& Feigelson, E. D. 2000, preprint

Mannings, V., \& Sargent, A. I. 1997, ApJ, 490, 792

Mathieu, R. D. 1994, ARAA, 32, 465

${ }^{1}$ IAU 200 Poster Proc. designates Birth and Evolution of Binary Stars, Poster Proceedings of IAU Symposium 200, ed. B. Reipurth \& H. Zinnecker 
Mazeh, T., Prato, L., \& Simon, M. 2000, IAU 200 Poster Proc. ${ }^{1}, 22$

Palla, F. \& Stahler, S. 1999, ApJ, 525, 772 (PS99)

Prato, L. A. 1998, PhD Thesis, SUNY-SB

Quast, G. R. et al. 2000, IAU 200 Poster Proc. ${ }^{1}, 28$

Reipurth, B. et al. 2000, IAU 200 Poster Proc. ${ }^{1}, 25$

Siess, L., Dufour, E., \& Forestini, M. 2000, A\&A, in press

Simon, M. 1996, ApJ, 469, 890

Simon, M., Dutrey, A., \& Guilloteau, S. 2000, ApJ, submitted

Steffen, A. et al. 2000, IAU 200 Poster Proc. ${ }^{1}, 19$

Swenson, F. J. et al. 1994, ApJ, 425, 286

Thiébaut, E. 1995, A\&A, 304, L17

Torres, G., Stefanik, R. P., \& Latham, D. W. 1997, ApJ, 485, 167

Torres, C. A. O. et al. 2000, IAU 200 Poster Proc. ${ }^{1}, 118$

van den Ancker, M. E. et al. 1998, A\&A, 330, 145

White, R. J. et al. 1999, ApJ, 520, 811

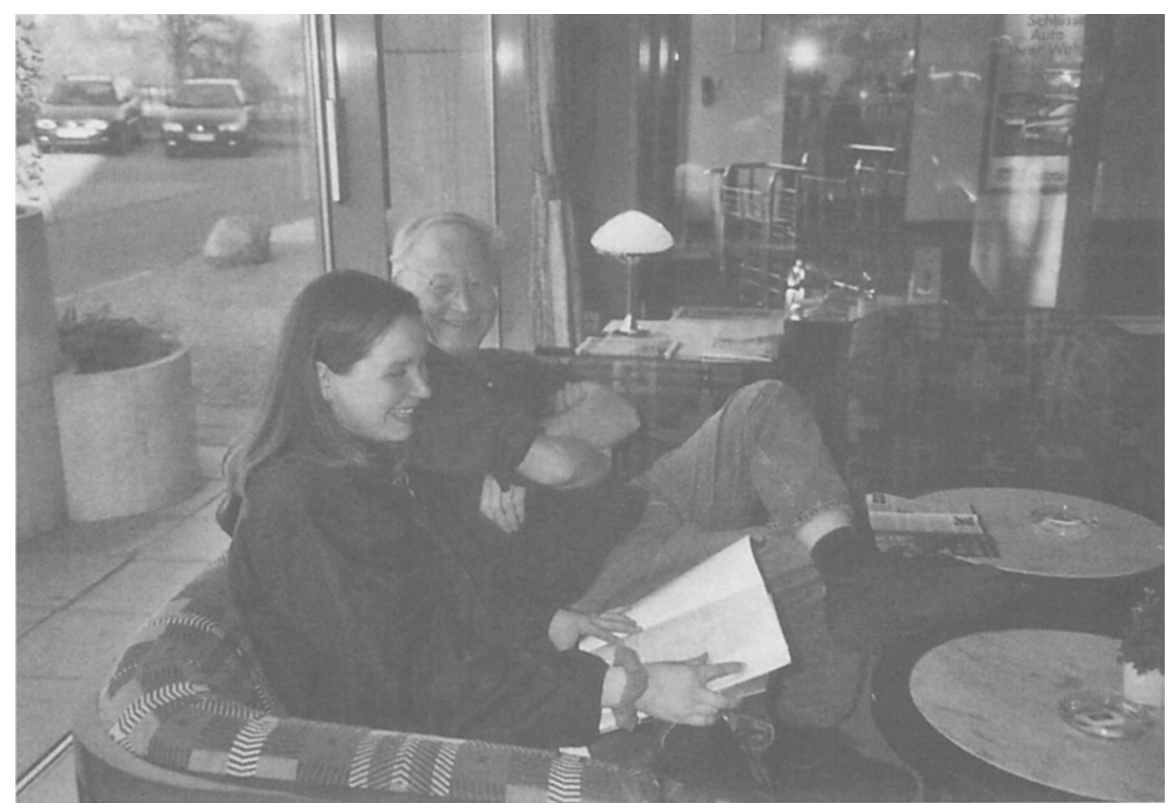

Mike Simon and his student Tracy Beck 